\title{
ECG Compression for Remote Healthcare Systems using Selective Thresholding based on Energy Compaction
}

\author{
Dwaipayan Biswas, Evangelos B. Mazomenos, Koushik Maharatna \\ School of Electronics and Computer Science \\ University of Southampton, SO17 1BJ, U.K \\ Email:db9g10,ebm,km3@ecs.soton.ac.uk
}

\begin{abstract}
This paper presents a wavelet-based low-complexity Electrocardiogram (ECG) compression algorithm for mobile healthcare systems, in the backdrop of real clinical requirements. The proposed method aims at achieving good trade-off between the compression ratio (CR) and the fidelity of the reconstructed signal, to preserve the clinically diagnostic features. Keeping the computational complexity at a minimal level is paramount since the application area we consider is that of remote cardiovascular monitoring, where continuous sensing and processing takes place in low-power, computationally constrained devices. The proposed compression methodology is based on the Discrete Wavelet Transform (DWT). The energy packing efficiency of the DWT coefficients at different resolution levels is analysed and a thresholding policy is applied to select only those coefficients which have significant contribution to the original signal total energy. The proposed methodology is evaluated on normal and abnormal ECG signals extracted from the MIT-BIH database and achieves an average compression ratio of 16.5:1, an average percent root mean square difference of 0.75 and an average cross correlation value of 0.98 .
\end{abstract}

\section{Motivation}

Continuous ascendancy of Cardiovascular Diseases (CVD) - the number one cause of death (30\% of the global total of all deaths) according to World Health Organization (WHO) - have put current healthcare systems worldwide, under serious strain in terms of quality of care delivery and its associated cost [1]. A significant drawback of current healthcare systems has been the post-incident strategy they follow; that is, healthcare actions are delivered after an occurrence of a critical event. In recent years, this has been countered with the development of next-generation 24-hour remote CVD monitoring and management systems, facilitated by breakthrough advances in Wireless Sensor Networks (WSN) technology, capable to monitor the patients' vital signs continuously in a nomadic environment.

A number of battery powered wireless sensors are considered to be placed on the patient's body in order to capture the vital clinical information and transmit data to a centralized service for further analysis allowing remote patient's monitoring and disease prognosis. The clinical feature extraction

This work was supported by E.U. ARTEMIS Joint Undertaking under the Cyclic and person-centric Health management: Integrated appRoach for hOme, mobile and clinical eNvironments - (CHIRON) Project, Grant Agreement \# 2009-1-100228. and information fusion processes, necessary to attain clinical diagnosis, are computationally intensive tasks and thus are typically carried out in the main-frame computational facilities for prolonging the battery life of the body-worn sensors. However, a long-term sustainable operation of this system is severely affected by the significant energy expenditure required by the radio front-end for supporting continuous data transmission [2].

In contrast to this, the emerging trend in the field of telemedicine, advocates that the capturing of the vital data and the feature extraction and information fusion operations are carried out at the on-body battery powered WSN nodes themselves, while transmission of the clinically relevant parameter to the centralized facility takes place only when required. In this approach the raw data is stored on the node itself and can be transmitted, on the clinician's demand or at preset intervals, in burst and therefore negate the necessity for continuous transmission while fully maintaining the notion of continuous monitoring. This system requires an efficient compression technique for storing the raw data as well as the clinically extracted cardiac features on the sensor node and for transmitting them to the remote server on request.

The application scenario that we consider is that of remote CVD monitoring applications, where capturing the patients' ECG signal, with considerably high-sampling frequencies $(250-500 \mathrm{~Hz})$, is paramount. The on-body storage requirements coupled with the energy expenditure during processing and transmission, necessitates the development of a lowcomplexity compression methodology that is less computationally demanding and thus implementable on ultra low-power hardware suitable for integration in a WSN node in the form of a dedicated ASIC. This prompts us to investigate the tradeoff between the degree of compression and the level of fidelity of the reconstructed signal at the clinician's end.

The main aim of ECG compression is to minimize the number of samples required to store or transmit, without losing the significant signal information, allowing for a nearaccurate reconstruction, which is a requirement for proper clinical diagnosis. Typical compression methodologies can be broadly classified as lossy or lossless. Lossy compression techniques are mainly applicable in image or video compression applications, whereas when dealing with critical vital sign data 
like ECG, maintaining the fidelity of the signal during reconstruction is deemed to be more important. Therefore lossless and near-lossless compression techniques are considered more applicable.

Lossless ECG compression methods explored in recent years mainly include block-sorting transformations of contextdepth one (linear order transformation (LOT)) or contextdepth " $n$ " (BurrowsWheeler transformation (BWT)), followed by Move-to-Front (MTF) and a suitable arithmetic coder [3]. However, these methods result in low compression ratios, which resulted in various lossy techniques to be considered for ECG compression.

In the relevant literature [4], [5] and [6] lossy ECG compression algorithms are grouped into three distinct categories; namely a) Direct Methods - Heuristic algorithms like AZTEC, FAN algorithm, TP, CORTES, SAPA and Entropy Coding, b) Transform Based Methods in which the original signal is transformed to a new domain where compression is performed, for example DCT, STFT, KLT and the more recently Wavelet Transform (WT). Most of these methods, transform the signal into a number of coefficients which represent the contribution of each frequency component in the signal's total energy. Obviously a considerable amount of coefficients will have minimal energy contribution and can be discarded resulting in a smaller-compressed amount of coefficient required to reconstruct the signal with acceptable accuracy. c) Parametric methods like Linear prediction.

WT based methods, are lossy compression techniques but yield a significantly high degree of compression. In this paper, we explore a computationally efficient compression methodology, using Discrete Wavelet Transform (DWT) combined with a thresholding technique based on the investigation of the Energy Packing Efficiency (EPE) of the resulting DWT coefficients. The compressed coefficients are then used to reconstruct the ECG signal using the Inverse Discrete Wavelet Transform (IDWT). We establish the fidelity of the reconstructed signal by applying the Time Domain Morphology [7] for extraction of the clinical features from the original ECG signal as well as on the reconstructed ECG signal in addition to the endorsement by practising clinicians.

The choice of the Wavelet function is of utmost importance since it decides on the computational complexity of the DWT process. For ECG compression, Daubechies, Biorthogonal and Symlet mother wavelets have been previously used [8] and [6]. Nevertheless the mathematical complexity of the aforementioned mother functions does not favor the design of a low-power architecture, let alone any ASIC implementation. Hence, we select the Haar function, the simplest in terms of complexity of the wavelet functions, for our DWT decomposition. Although this function has its own limitations, we hypothesized that it still may be sufficient for the present purpose of ECG compression. Primary aim of this work is to investigate to what extent Haar DWT analysis combined with multiple resolution level EPE based coefficient selection, can result in an efficient ECG compression scheme that achieves a good trade-off between, computational complexity, compression ratio and fidelity of the reconstructed signal. In our analysis, the proposed compression algorithm has been tested on a large number of records from the MIT-BIH arrhythmia database and also on 12 digitised Paper ECG signals supplied by the Southampton General Hospital's Cardiology Department (SGHCD). In all cases the reconstructed signal has been endorsed by expert cardiologists from SGHCD.

The rest of the paper is structured as follows: Section II presents a theoretical background of DWT and compression parameters, we describe our exploration on EPE based threshold selection in Section III. Section IV is devoted on the validation of the compression methodology and simulation results. The conclusions are drawn in Section V.

\section{TheORETICAL BACKGROUND}

\section{A. DWT}

DWT is used to analyse the signals in both time and frequency domain making it suitable for the analysis of timevarying non-stationary signals such as ECG. The frequency and time localization property of DWT offer a great advantage since fewer basis functions are required to represent the signal to a given level of approximation [5]. In the proposed compression scheme, the multi-scale DWT decomposition is implemented as a cascade filter-bank structure (known as Mallat's Algorithm), illustrated in Fig1 featuring high and low-pass filters. Downsampling is performed after filtering, to remove redundancy. As DWT transforms the original signal into multi-resolution bands each having a reduced number of coefficients, it can be effectively used for compressing the samples. The output of the high pass filters (H1(z)) provides the detailed WT coefficients (cD_lx) at the $2^{\mathrm{x}}$ scale, while the approximate WT coefficients (cA_lx) are obtained from the output of the low-pass filters ( $\mathrm{HO}(\mathrm{z}))$. The higher the scale, the higher the temporal resolution of low frequency components become. Thus high frequency components are expected to be represented with higher resolution in the lower scales $\left(2^{1}, 2^{2}\right)$ while low frequency components in the higher analysis scales $\left(2^{4}, 2^{5}\right)$

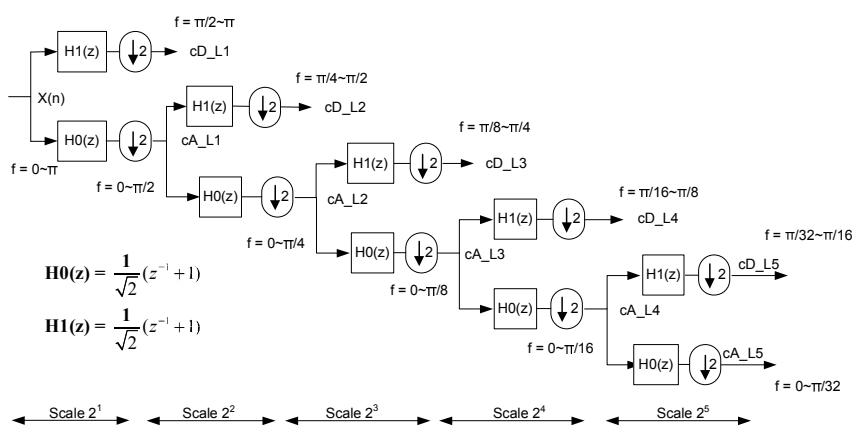

Fig. 1. Cascade filter-bank Implementation of DWT

\section{B. Compression Performance Parameters}

The performance of the compression methodology is measured in terms of the following parameters mentioned below: 
a) Compression Ratio (CR) - It is a ratio of the number of samples representing the original ECG signal and the compressed ECG signal and is expressed as :

$$
C R=\frac{N_{b c}}{N_{a c}}
$$

where $N_{b c}$ and $N_{a c}$ are the number of samples in the compressed signal and the original ECG signal respectively.

b) Percent root mean square difference (PRD) - It is a measure of expressing the error or the distortion in the reconstructed signal as compared to the original ECG signal, and hence establishes the effectiveness of the compression methodology and is expressed as :

$$
\operatorname{PRD}\left({ }^{\prime} \%^{\prime}\right)=\sqrt{\frac{\sum_{n=1}^{N_{i}}(x(n)-\hat{x}(n))^{2}}{\sum_{n=1}^{N}(x(n))^{2}}} \times 100
$$

where $x(n)$ and $\hat{x}(n)$ are the original and the reconstructed ECG signal respectively.

c) Cross correlation (CC) - It is used to evaluate the similarity between the original and the reconstructed ECG signal and establishes the fidelity of the reconstructed signal, it is expressed as :

$$
C C=\frac{\frac{1}{N} \sum_{i=1}^{N}\left(x_{i}-\bar{x}\right)\left(\hat{x}_{i}-\overline{\hat{x}}\right)}{\sqrt{\frac{1}{N} \sum_{i=1}^{N}\left(x_{i}-\bar{x}\right)^{2}} \sqrt{\frac{1}{N} \sum_{i=1}^{N}\left(\hat{x}_{i}-\overline{\hat{x}}\right)^{2}}}
$$

where $x$ and $\hat{x}$ are the original and the reconstructed ECG signal respectively and $\bar{x}$ and $\bar{x}$ are their respective means.

\section{Energy Compaction and Thresholding}

After the ECG signal is decomposed through DWT, selective thresholding is applied in its coefficients, in view of their energy compaction, at the respective resolution levels. The energy contribution of each wavelet decomposition subband with respect to the original signal as well as the other coefficients is analysed using EPE [9]. This is a quantitative figure, expressed in terms of percentage, which represents a measure of the energy content of the coefficients of a certain sub-band after thresholding with respect to the total energy in that sub-band before thresholding.

$$
\operatorname{EPE}\left({ }^{\prime} \%{ }^{\prime}\right)=\sqrt{\frac{\sum_{n=1}^{L_{i}}(c(n))^{2}}{\sum_{n=1}^{L}(c(n))^{2}}} \times 100
$$

where $L_{i}$ and $L$ are the number of coefficients in the $i_{t h}$ subband after and before thresholding respectively.

The performance, in terms of compression ratio, of the compression methodology and the fidelity of the reconstructed signal also have a direct relationship with the resolution scale(s) of DWT that we choose for compressing the signal. The higher the resolution level the smaller the number of DWT coefficients, due to subsampling. It is then normal to achieve higher CR and PRD values by focusing on the high $\left(2^{4}, 2^{5}\right)$ resolution scales. Conversely, compressing the ECG using the low resolution scales $\left(2^{2}, 2^{3}\right)$ results in low CR and PRD but achieves a more accurate reconstruction of the original signal in terms of the cross correlation between the original signal and the reconstructed signal. To present a complete investigation on the trade-off between CR and accuracy that can be achieved, we decompose the ECG signal using DWT upto resolution level $2^{5}$ and we study the energy content variation among the detail and approximate coefficients at various resolution levels while also exploring various threshold ranges to be selected on the coefficients at the respective dyadic scales. This analysis allows to select the resolution level(s) that achieve the optimum trade-off between CR and reconstruction fidelity. Resolution scale $2^{1}$ is disregarded in our investigation since it is considered to be dominated from the high frequency noise components.

The threshold value also influences the level of data compression, since a high threshold can result in high $\mathrm{CR}$ but poor signal fidelity after reconstruction and on the contrary, a small threshold would produce low data reduction but high signal fidelity. The optimal choice of the threshold value is a key point of ECG data compression. To determine the threshold level in each resolution level, the energy packing efficiency (EPE) [5], [10] of the coefficients in each sub-band is calculated. Since DWT transforms the signal to multi-resolution bands, the one that has the lowest frequency components, the approximation band, contains high-amplitude coefficients, thus carrying the majority of the original ECG signal energy. Hence, we have selected not to threshold the approximate coefficients and consider all of them in the compressed signal [8]. The detail coefficients, the output of the high-pass filter, are of lesser magnitude which signifies that they carry a smaller percentage of the signal's energy. These are also vital and need to be used, in combination with the approximate coefficients, for reconstruction however we choose to store only the detail coefficient which exceed the EPE based threshold, thus represent component with significant energy and disregard the remaining one's.

Selective thresholding is achieved by detecting the maximum and minimum value of each detail sub-band and considering only those coefficients which have a higher or lower magnitude from a certain percentage of the maximum or the minimum value respectively. Different to various other methods in the relevant literature [9] that only consider the maximum value, to define the threshold, we additionally consider the minimum coefficient value since it also plays an important role in retaining those samples which represent the characteristic waves (like the S-wave) of an ECG signal which are negative, compared to the isoelectric line, deflections. In our exploration, we avoid steps that involve any real divisions or multiplications and we only employ divisions/multiplications that can be represented as a dyadic 
fraction (to a power of 2) and implemented in the form of hard-wired shifters. It is our belief that following this approach the resulting compression algorithm can have a potential for low-power architecture implementation.

After the selective thresholding process is completed, the remaining coefficients represent the compressed version of the original ECG sample. The compressed signal can then be transmitted and reconstructed with IDWT at the clinician's end, using only the approximate and thresholded detail coefficients.

\section{Selective Thresholding BASED ON EPE}

We decided to explore four different sets of thresholding and compressing methodologies, based on an analytical study of the EPE values of the approximate and detail coefficients at resolution levels $2^{3}$ and $2^{5}$. To quantify the percentage of energy contained in these two resolution scales, we average the EPE values of 17 ECG signals tested from MIT-BIH arrhythmia database. The EPE results are provided in Table I.

The significant bands of coefficients, produced as a result of the DWT decomposition and considered for our case are : cA_15 (level 5 approximation coefficient), cD_15 (level 5 detail coefficient) and cD_14 (level 4 detail coefficient), cA_13 (level 3 approximation coefficient), cD_13 (level 3 detail coefficient), cD_12 (level 2 detail coefficient) and cD_11 (level 1 detail coefficient).

\begin{tabular}{|l|l|}
\hline EPE for different sub-bands & EPE values(\%) \\
\hline \hline EPE_D1 & $0.00267 \%$ \\
\hline EPE_D2 & $0.00925 \%$ \\
\hline EPE_D3 & $0.02545 \%$ \\
\hline EPE_A3 & $99.96262 \%$ \\
\hline EPE_D1D2D3 & $0.03738 \%$ \\
\hline EPE_D3_D1D2D3 & $60.89235 \%$ \\
\hline EPE_D2_D1D2D3 & $29.31935 \%$ \\
\hline EPE_D1_D1D2D3 & $10.80832 \%$ \\
\hline
\end{tabular}

TABLE I

EPE VALUES FOR APPROXIMATE AND DETAIL SUB-BANDS IN RESOLUTION LEVEL $2^{3}$

1) Analysis of $2^{3}$ scale coefficients: EPE_A3 represents the energy content of the samples in the level 3 approximation band as compared to the the energy of the original signal and it accounts for $99.96 \%$ of the signal energy. The combined energy content of the detail coefficients of levels 3,2 and 1 represented by EPE_D1D2D3 is $0.037 \%$. As discussed in the previous section, the cA_13 coefficients are left without thresholding due to their high energy content and then we separately select distinct threshold values for each of the detail level coefficients. The energy contribution of the detail coefficients to the total energy of the signal reduces from that in detail level 3 to level 1 as illustrated by the values EPE_D3, EPE_D2, and EPE_D1. Before thresholding the detail coefficients, we investigate their energy content at each level with respect to the combined detail energy.

The energy content of $\mathrm{cD}$ 13 to the combined detail level energy, EPE_D3_D3D2D1 is $60.89 \%$, similarly that of cD_12, EPE_D2_D3D2D1 is $28.29 \%$ and for cD_11, EPE_D1_D3D2D1 is $10.80 \%$. The selection of thresholds for the respective levels should ensure that maximum coefficients are chosen from cD_13 whereas the minimum number of coefficients are chosen from cD_11.

Experimentally, we choose the threshold values as $32 \%$ and $64 \%$ for selecting the coefficients of cD_13 and cD_12 coefficient and we completely discard the cD_11 coefficients since they have a minimum energy content. We select those coefficients from cD_13 and cD_12 which are greater than $32 \%$ and $64 \%$ of the maximum value and less than $32 \%$ and $64 \%$ of the minimum value of the sample size respectively.

The choice of the threshold value is guided by the following two observations. Firstly, the number of samples in cD_12 is double the number of coefficients in $\mathrm{cD} \_13$ and secondly the energy content of $\mathrm{cD}_{-} 12$ with respect to the total detail sub-band energy (EPE_D2_D1D2D3) is approximately half of the energy content of cD_13 (EPE_D3_D1D2D3). The chosen threshold value $(32 \%, 64 \%)$ at the resolution levels $2^{3}$ and $2^{2}$ is in the ratio of $1: 2$, which in effect ensures that we select more samples with significant magnitude from cD_13 than $\mathrm{cD}_{-} 12$. The energy contribution of the compressed sample size of cD_13 and cD_12 after thresholding to their original sample size is shown in Table II below :

\begin{tabular}{|l|l|}
\hline EPE for different sub-bands & EPE values(\%) \\
\hline \hline EPE_D3_comp & $85.10 \%$ \\
\hline EPE_D2_comp & $61.85734 \%$ \\
\hline EPE_D1_comp & 0 \\
\hline
\end{tabular}

TABLE II

EPE VALUES FOR COMPRESSED DETAIL COEFFICIENTS OF RESOLUTION LEVEL $2^{3}, 2^{2}$ AND $2^{1}$

The high EPE values of the compressed coefficients ensure that we preserve a majority of the significant detail coefficients. This sums up our first method of exploration (Method 1) at $2^{3}$ scale.

2) Analysis of $2^{5}$ scale coefficients: As discussed before, an improvement in the CR is expected if coefficients from higher level of DWT decomposition are used, thus we also analyse the decomposed coefficients of resolution level $2^{5}$. The average EPE values of the 17 ECG signals are listed in Table III below.

\begin{tabular}{|l|l|}
\hline EPE for different sub-bands & EPE values(\%) \\
\hline \hline EPE_D4 & $0.05621 \%$ \\
\hline EPE_D5 & $0.09113 \%$ \\
\hline EPE_A5 & $99.81528 \%$ \\
\hline EPE_D1D2D3D4D5 & $0.18472 \%$ \\
\hline EPE_D5_D1D2D3D4D5 & $41.74150 \%$ \\
\hline EPE_D4_D1D2D3D4D5 & $31.93224 \%$ \\
\hline EPE_D3_D1D2D3D4D5 & $14.91434 \%$ \\
\hline EPE_D2_D1D2D3D4D5 & $7.98171 \%$ \\
\hline EPE_D1_D1D2D3D4D5 & $3.43019 \%$ \\
\hline
\end{tabular}

TABLE III

EPE VALUES FOR APPROXIMATE AND DETAIL SUB-BANDS IN RESOLUTION LEVEL $2^{5}$

Inspired from our threshold calculations performed in the 
preceding subsection for the $2^{3}$ scale, we keep all the coefficients of cA_15 since it comprises of samples having the highest energy content (EPE_A5 $=99.81528 \%$ ) with respect to the original signal and subsequently, we decide on three ways of thresholding the detail coefficients. The threshold value represents the energy content of the detail coefficients across all resolution levels and have been experimentally deduced for each method as presented below :

a) Method 2 - selecting a threshold of $8 \%, 16 \%$ on the maximum and minimum value of the coefficients of $\mathrm{cD}$ - 15 and cD_14 respectively, thus completely neglecting the coefficients of cD_13, cD_12 and cD_11

b) Method 3 - selecting a threshold of $8 \%, 16 \%, 64 \%$ on the maximum and minimum value of the coefficients of $\mathrm{cD}$ - 15 , cD_14 and cD_13 respectively, thus completely neglecting the coefficients of cD_12 and cD_11

c) Method 4 - selecting a threshold of $8 \%, 16 \%, 64 \%$ and $64 \%$ on the maximum and minimum value of the coefficients of cD_15, cD_14 and cD_13, cD_12 respectively, thus completely neglecting the coefficients of cD_l1.

The energy contribution of the compressed (after thresholding) sample size of the detail coefficients with respect to their original signal is shown in Table IV below :

\begin{tabular}{|l|l|}
\hline EPE for different sub-bands & EPE values(\%) \\
\hline \hline EPE_D3_comp & $99.14362 \%$ \\
\hline EPE_D4_comp & $95.36816 \%$ \\
\hline EPE_D3_comp & $66.51193 \%$ \\
\hline EPE_D2_comp & $61.8573 \%$ \\
\hline EPE_D1_comp & 0 \\
\hline
\end{tabular}

TABLE IV

EPE VALUES FOR COMPRESSED DETAIL COEFFICIENTS OF RESOLUTION LEVEL $2^{5}, 2^{4}, 2^{3}, 2^{2}$ AND $2^{1}$

The discarded insignificant samples in the detail sub-bands are set to zero. Modified run length encoding is used to count the number of zeroes and their relevant positions which helps in reconfiguring the detail sub-band signal. Inverse DWT is used to reconstruct the original signal from the compressed coefficients. Depending on which compression method is followed, IDWT might run from the $3^{\text {rd }}$ or the $5^{\text {th }}$ level upto the original resolution scale for fully reconstructing the original ECG sample.

\section{RESUlts AND Discussion}

In this section we provide and discuss analytical results of our compression/reconstruction methodology. In our investigation, ECG excerpts from various databases were used. Both ambulatory ECG databases (MIT-BIH arrhythmia database) and standard 15-lead ECG (PTB database (PTBDB)) [11]. Finally 12 paper ECG signals from the SGHCD database, captured through the standard 12-lead ECG machine were used after being digitised. For our experiment purpose we fix up a segment length of 2048 samples to work with. The signal length is of vital importance since a large sample size increases the variance in the sub-band signal's distortion and affects the performance of the compression methodology. For enabling comparison to other compression approaches, we present here the average performance of the four compression methods on the records 100,101,102,103,116, 117, 200, 201, 202, 207, $210,215,220,221,223,231$ and 232 having a sampling frequency of $360 \mathrm{~Hz}$ from MIT-BIH arrhythmia database in Table V.

\begin{tabular}{|l|l|l|l|l|}
\hline Parameters & Method 1 & Method 2 & Method 3 & Method 4 \\
\hline \hline CR & 7.143880044 & 18.25359633 & 16.27602544 & $19.38809456 \%$ \\
\hline PRD (\%) & 0.587429996 & 0.905119604 & 0.753811454 & $1.220041521 \%$ \\
\hline CC & 0.95392222 & 0.95274911 & 0.974509213 & $0.937217502 \%$ \\
\hline
\end{tabular}

TABLE $\mathrm{V}$

COMPARATIVE VALUES FOR CR, PRD AND CC FOR THE FOUR METHODS OF COMPRESSION

The data from Table $\mathrm{V}$ clearly reflects the effectiveness of Method 3 which demonstrated a low PRD and high CR and $\mathrm{CC}$ values, therefore ensuring high fidelity of the reconstructed ECG signal. A comparative study of the performance of our algorithm used in Method 3 along with other compression techniques for record 117 of MIT-BIH arrhythmia database as reported in [6] is presented in Table VI.

\begin{tabular}{|l|l|l|}
\hline Algorithm & CR & PRD (\%) \\
\hline \hline SPIHT & $8: 1$ & $1.18 \%$ \\
\hline Hilton & $8: 1$ & $2.6 \%$ \\
\hline Djohn & $8: 1$ & $3.9 \%$ \\
\hline CORTEC & $4.8: 1$ & $7 \%$ \\
\hline WT \& Huffman & $13.57: 1$ & $4.87 \%$ \\
\hline Ebrahimzadeh,A [6] & $13.92: 1$ & $0.97 \%$ \\
\hline Method 3 & $18.96: 1$ & $1.21 \%$ \\
\hline
\end{tabular}

TABLE VI

COMPARATIVE PERFORMANCE OF THE PROPOSED ALGORITHM WITH OTHER ECG COMPRESSION ALGORITHMS

The original and the reconstructed ECG signal for records 117,221 and 232 from the MIT-BIH arrhythmia database are shown in Fig 2.

To additionally verify the effectiveness of our method, we use the previous three ECG samples with our TDMG feature extraction algorithm [7]. The TDMG algorithm operates on a single heartbeat and extracts the temporal positions of the important clinical parameters (wave boundaries, peak positions). For each record we select the same heartbeat (PQRST complex) from both the original and reconstructed signal and compare the TDMG algorithm's output. The results are given in terms of the sample number where we detect the respective ECG feature and are illustrated in Table VII. It is clear that almost all parameters, from the three records, are detected within 5 samples. Thus it is evident from Tables VI and VII that we have been able to conserve the essential clinical features in pursuit of achieving a high degree of compression.

\section{CONCLUSiOns}

This work addresses the major challenges of the modern remote monitoring system wherein the feature extraction and disease classification takes place at the sensor nodes itself 

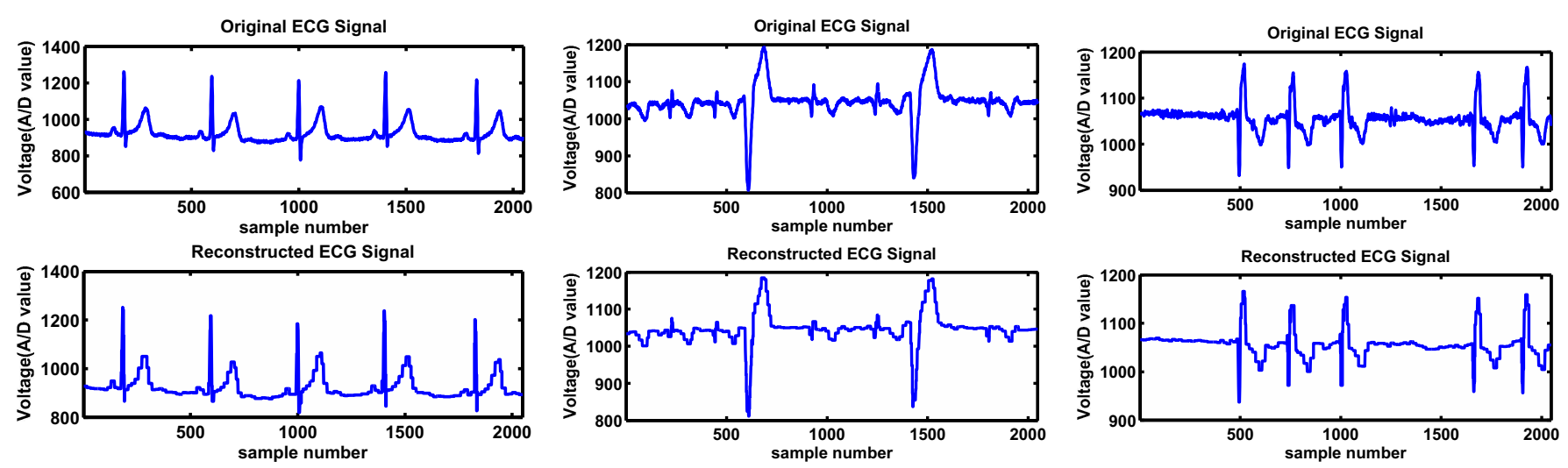

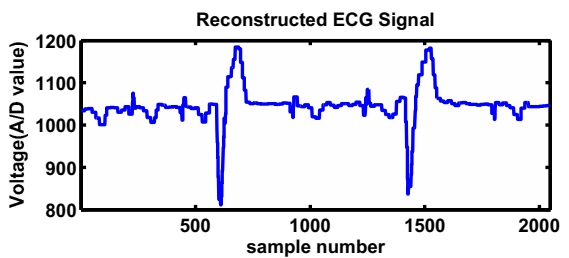

(b)

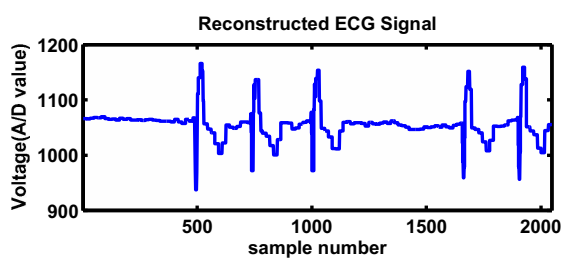

(c)

Fig. 2. MIT-BIH records 117(a), 221(b), 232(c) original signal and after reconstruction

\begin{tabular}{|c|c|c|c|c|c|c|c|c|c|c|}
\hline Record & Feature & $P_{\text {on }}$ & $P_{\text {peak }}$ & $P_{\text {off }}$ & $Q R S_{\text {on }}$ & $R_{\text {peak }}$ & $Q R S_{\text {off }}$ & $T_{\text {on }}$ & $T_{\text {peak }}$ & $T_{\text {off }}$ \\
\hline \hline \multirow{3}{*}{117} & original & 116 & 137 & 156 & 163 & 183 & 211 & 232 & 288 & 322 \\
\cline { 2 - 12 } & reconstructed & 121 & 138 & 154 & 163 & 183 & 213 & 235 & 289 & 327 \\
\cline { 2 - 11 } 221 & original & 66 & 87 & 102 & 126 & 168 & 194 & 218 & 254 & 284 \\
\cline { 2 - 11 } & reconstructed & 75 & 92 & 108 & 126 & 168 & 194 & 217 & 252 & 282 \\
\cline { 2 - 11 } 232 & original & 45 & 63 & 91 & 101 & 136 & 161 & 190 & 231 & 277 \\
\cline { 2 - 11 } & reconstructed & 42 & 59 & 73 & 99 & 137 & 163 & 183 & 234 & 280 \\
\hline
\end{tabular}

TABLE VII

Feature Extraction PERformance Results on MIT-BIH Records

placed on the patient's body thus creating a need for a lowcomplexity compression-reconstruction methodology. Here we performed a comprehensive investigation of the performance of a compression strategy based on the Haar DWT and on EPE selection. Due to the inherent linearity property of the Haar function, the generalized DWT architecture can be realised wherein the coefficients can be computed directly from the incoming ECG signal on the fly, by using simple additions and subtractions using ripple carry adders which in-effect reduces the complexity and throughput time of calculating the DWT coefficients. We decompose the signal upto $2^{5}$ since we have experimentally verified that a vast majority of ECG frequency components is contained within these resolution scales.

Four methods of selecting the coefficients have been explored and their corresponding $\mathrm{CR}$, PRD and CC values have been used to access their performance. The compression algorithm along with the threshold percentages have been developed in view of an architecture implementation and can be realised as an ASIC. With an average CR, PRD and CC of $16.27: 1,0.75$ and 0.975 respectively we have been able to find the right trade-off between compression ratio and maintaining the fidelity of the reconstructed ECG signal. From the results, Method 3 stands out, as it effectively balances the level of compression (CR value) and the fidelity of the reconstructed signal (PRD value). Although, with signals having different sampling frequencies from PTBDB and SGHCD there is a change in the values of the compression performance parameters we conclude that this method still satisfies our objective for mobile healthcare applications. We further solidify our argument by demonstrating that the compression process is capable of resulting in a reconstructed signal that maintains the same morphological patterns and clinical parameters as the original one.

\section{REFERENCES}

[1] Frost \& Sullivan, "Preparing for an aging society: challengers faced by healthcare system in european union, japan and united states," 2009. [Online]. Available: http://www.frost.com/

[2] R. Balani, "Energy consumption analysis for bluetooth, wifi and cellular networks," University of California at Los Angeles, Tech. Rep., 2007.

[3] Z. Arnavut, "Ecg signal compression based on burrows-wheeler transformation and inversion ranks of linear prediction," IEEE Trans. Biomed. Eng., vol. 54, no. 3, pp. 410 -418, Mar 2007.

[4] S. Jalaleddine, C. Hutchens, R. Strattan, and W. Coberly, "Ecg data compression techniques-a unified approach," IEEE Trans. Biomed. Eng., vol. 37, no. 4, pp. 329 -343, Apr 1990.

[5] M. Abo-Zahhad and B. Rajoub, "Ecg compression algorithm based on coding and energy compaction of the wavelet coefficients," in IEEE ICECS, vol. 1, 2001, pp. $441-444$ vol.1.

[6] A. Ebrahimzadeh and M. Azarbad, "Ecg compression using wavelet transform and three-level quantization," in IDC, Aug 2010, pp. 250 254.

[7] E. Mazomenos, T. Chen, A. Acharyya, A. Bhattacharya, J. Rosengarten, and K. Maharatna, "A time-domain morphology and gradient based algorithm for ECG feature extraction," in IEEE ICIT, 2012.

[8] B. Rajoub, "An efficient coding algorithm for the compression of ecg signals using the wavelet transform," IEEE Trans. Biomed. Eng., vol. 49, no. 4, pp. $355-362$, Apr 2002.

[9] M. Abo-Zahhad, S. Ahmed, and A. Al-Shrouf, "Electrocardiogram data compression algorithm based on the linear prediction of the wavelet coefficients," in IEEE ICECS, vol. 1, 2000, pp. 599 -603 vol.1.

[10] M. Hossain, T. Aziz, and M. Haque, "Ecg compression using multilevel thresholding of wavelet coefficients," in ISSNIP 2008, Dec 2008, pp. $321-326$.

[11] A.L. Goldberger et. al., "Physiobank, physiotoolkit, and physionet components of a new research resource for complex physiologic signals," Circulation, vol. 101, no. 23, 2000 (June 13). 